\title{
Submitted: Prenatal diagnosis of congenital megalourethra Accepted: in the second trimester of pregnancy
} 29.03.2019

Published: 31.12.2019

\section{Keywords}

prenatal diagnosis, congenital megalourethra, ultrasonography, postmortem

\author{
Do Duy Anh¹, Ha To Nguyen², Simon Meagher ${ }^{3}$, Edward Araujo Júnior ${ }^{4}$ \\ ${ }^{1}$ Pham Ngoc Thach University, Ho Chi Minh City, Vietnam \\ ${ }^{2}$ Imaging Diagnostic Department, Tudu maternity Hospital, Ho Chi Minh City, Vietnam \\ ${ }^{3}$ Monash Ultrasound for Women, Melbourne, Australia \\ ${ }^{4}$ Department of Obstetrics, Paulista School of Medicine, Federal University of São Paulo \\ (EPM-UNIFESP), São Paulo-SP, Brazil \\ Correspondence: Prof. Edward Araujo Júnior, PhD, Rua Belchior de Azevedo, 156 \\ apto. 111 Torre Vitoria, São Paulo-SP, Brazil, CEP 05089-030; tel./fax: +55-11-37965944, \\ e-mail:araujojred@terra.com.br
}

DOI: $10.15557 / J o U .2019 .0045$

\begin{abstract}
Congenital megalourethra is abnormal dilation of the penile urethra due to aplasia of erectile tissue, leading to lower urinary tract obstruction. This condition should be considered when fetal penis with typical dilation is seen on prenatal scan. The dilated structure, however, can be easily missed or misinterpreted as the umbilical cord. Perinatal prognosis is poor due to direct consequences on urinary and respiratory systems, or due to severe associated anomalies. A large number of survived cases experience urinary sequelae and renal impairment. We report one case of fetal congenital megalourethra diagnosed at 17 weeks and 6 days of pregnancy.
\end{abstract}

\section{Introduction}

Congenital megalourethra is typically characterized by the aplasia of penile erectile tissue, leading to abnormal dilation of the anterior urethra without structural obstruction $^{(1,2)}$. This terminology was first used in 1955 to describe an infant with abnormally enlarged penis and worsening renal function ${ }^{(2)}$. In 1989, Benacerraf et al. ${ }^{(3)}$ diagnosed megalourethra for the first time on fetal ultrasound. Congenital megalourethra is a rare condition. The prevalence is not known since most studies were solely focused on case reports and pathological autopsies ${ }^{(1,4)}$.

\section{Case report}

A 31 year-old primigravid pregnant woman with unremarkable history was referred to the Tu Du Hospital, Vietnam, with diagnosis of fetal megacystis detected on routine sonography. Ultrasound scan at 17 weeks 6 days reported the following findings: echogenic kidneys, right pyelectasis
(3.6 mm), dilated right ureter (3.2-3.6 mm) (Fig. 1), left pyelectasis $(3.2 \mathrm{~mm})$, dilated urinary bladder $(33 \times 24 \mathrm{~mm})$ with keyhole sign (Fig. 2), dorsally curved penis with largely dilated penile urethra $(18 \times 5 \mathrm{~mm})$ (Fig. 3$)$. The amniotic fluid volume was normal. No other abnormalities were found. Based on such abnormal sonographic findings, including hydronephrosis, hydroureters, megacystis and dilated penile urethra, we came to a diagnosis of scaphoid congenital megalourethra. After parental counseling, termination of pregnancy proved the prenatal findings (Fig. 4).

\section{Discussion}

Congenital megalourethra belongs to a group of disorders called lower urinary tract obstruction (LUTO). The most common etiologies are structural obstructions such as posterior urethral valve and urethral atresia ${ }^{(5,6)}$. Obstruction in congenital megalourethra, however, is functional rather than structural, hence featuring a different pathogenesis. Malformation occurs early in pregnancy and disrupts the 


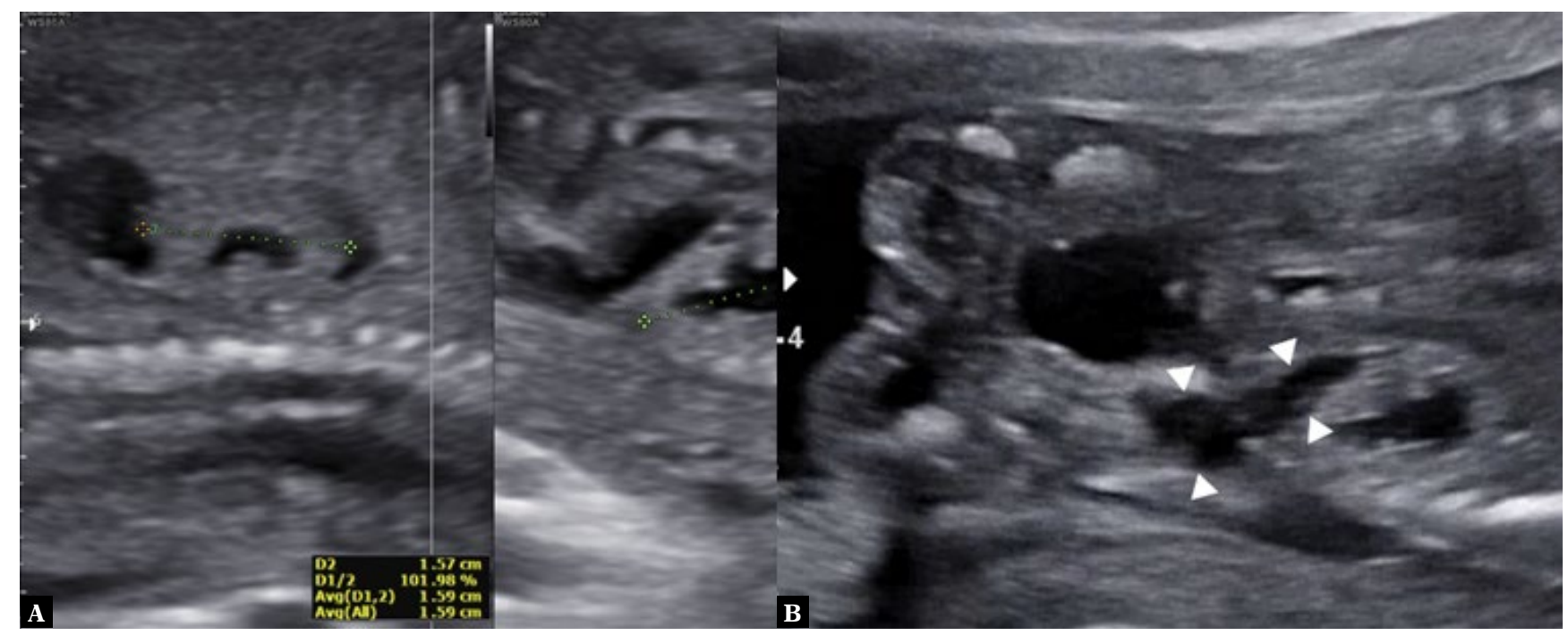

Fig. 1. A. Dilated right renal pelvis $\mathbf{B}$. dilated right ureter (arrowhead)

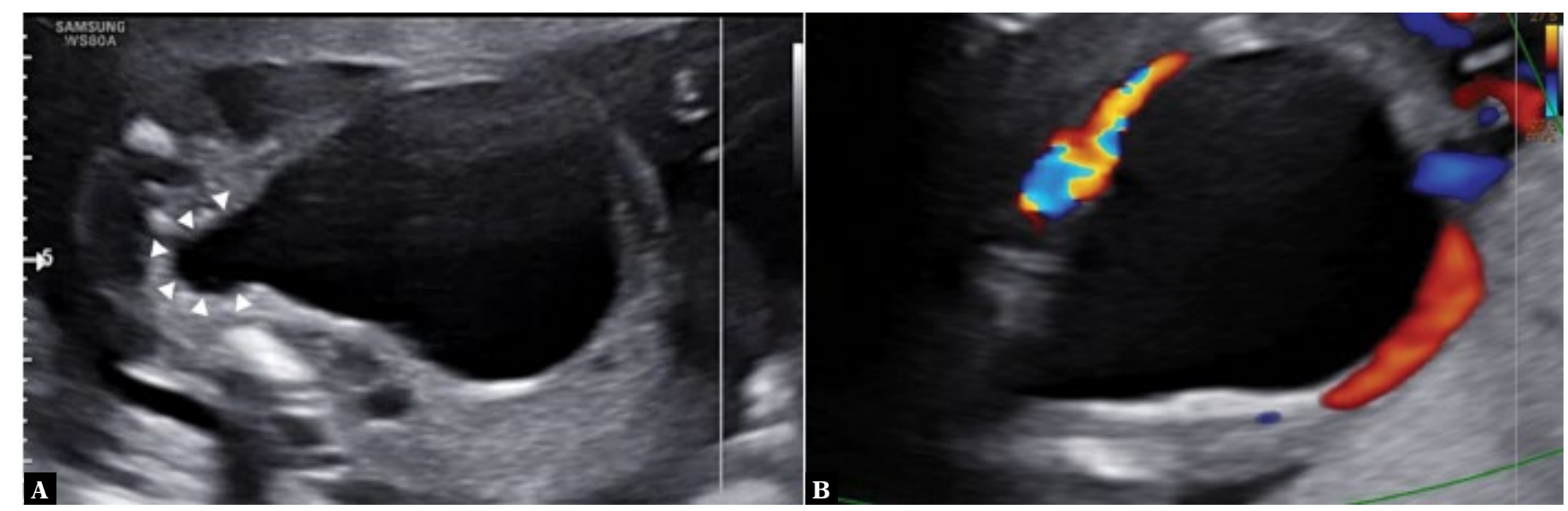

Fig. 2. A. Dilated urinary bladder with the keyhole sign (arrowhead) B. Two umbilical arteries on color flow Doppler mode

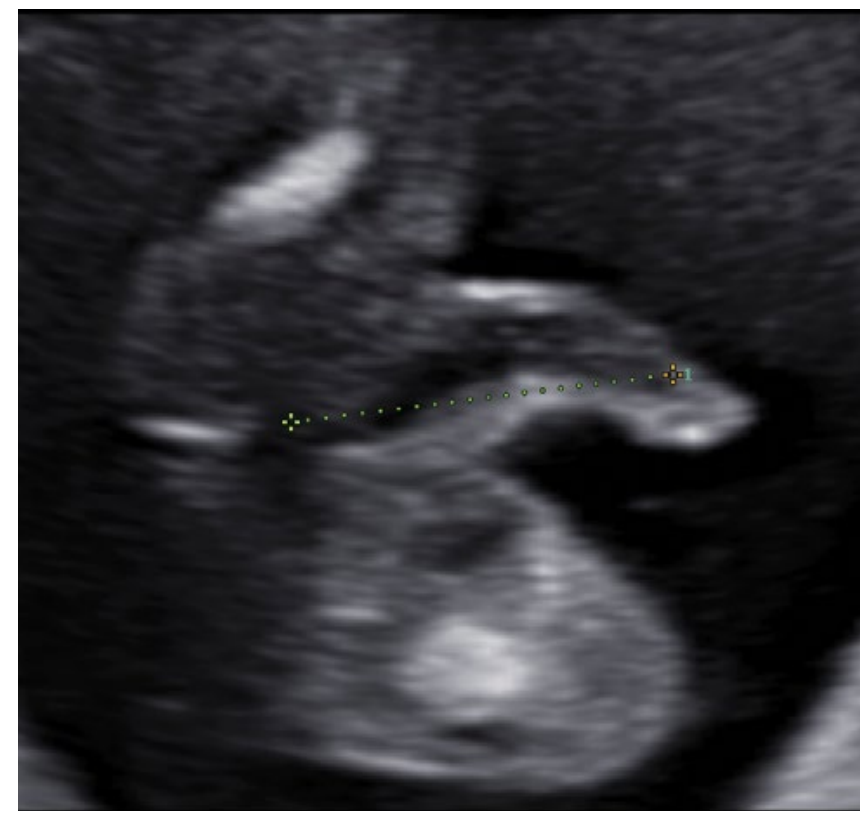

Fig. 3. Cystic penis with dilated penile urethra (the urethral meatus was clearly seen)

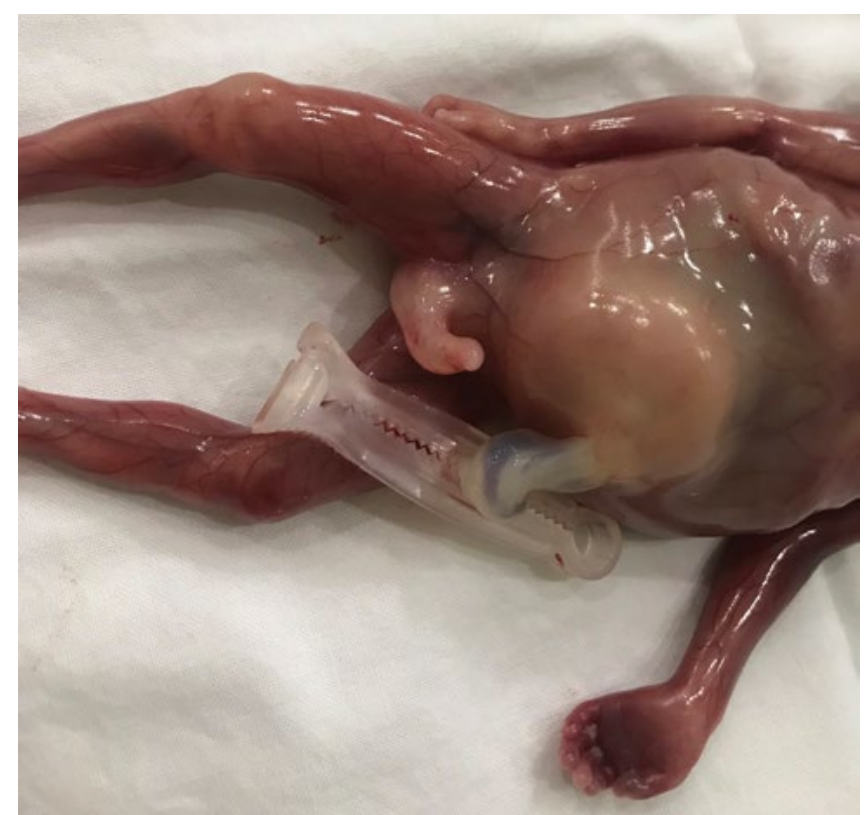

Fig. 4. Post-mortem examination confirmed an enlarged and dorsally curved penis 
combining process of mesoderm-derived structures including corpus spongiosum on the ventral side and corpus carvenosum on the dorsal side of the penis. This leads to aplasia of the erectile tissue, which normally supports the urethra on micturation. As a result, the penile urethra gradually dilates, causing urine stasis and retrograde obstruction ${ }^{(2,4,7)}$.

According to Dorairajan and Stephens, congenital megalourethra was morphologically divided into two types ${ }^{(2)}$ :

- Scaphoid type: $80 \%$ of cases. The corpus spongiosum is completely or partially absent while the corpus carvenosum is intact, making the penis curved dorsally.

- Fusiform type: $20 \%$ of cases. Complete aplasia of both corpus spongiosum and corpus carvenosum. The penile urethra is just covered by a thin layer of skin and connective tissue, making the penis a cystic rod-shaped structure. This type has much worse prognosis with high prevalence of associated anomalies.

All cases were diagnosed prenatally via ultrasound. Most diagnoses were made in the second trimester ( $86 \%)$, followed by third $(12 \%)$ and first trimester $(2 \%)^{(1)}$. The high rate of detection in the second trimester could be explained by the emergence of complications (megacystis, hydroureters, hydronephrosis, oligohydramnios) or detection of other associated anomalies ${ }^{(7)}$.

The rate of live births was reported to be about $60-70 \%{ }^{(1,2)}$. Most patients suffered from sequelae such as abnormally large penis, erectile and urinating dysfunction, recurrent urinary tract infection, renal failure, etc. ${ }^{(4)}$. Postnatal follow-up revealed that renal impairment rate was up to $42 \%$, with three prenatal risk factors including:

- oligohydramnios or anhydramnios,

- bilateral hydronephrosis, and

- small fetal age at birth ${ }^{(1)}$.

When congenital megalourethra is suspected on ultrasound, it is necessary to rule out other conditions of similar sonographic presentation, yet much higher prevalence.
Similar presentation of megacystis:

- Posterior urethral valve (57\%). Approximately $24 \%$ of congenital megalourethra cases were, however, accompanied by posterior urethral valve. This should be considered as a concomitant anomaly rather than the direct cause of dilated penile urethra. On the other hand, 12\% of isolated congenital megalourethra cases presented the "keyhole sign" on ultrasound, which is specific for posterior urethral valve or urethral atresia. When this sign is detected, a careful examination of the penile urethra to justify a correct diagnosis ${ }^{(1)}$;

- Urethra atresia (7\%);

- Prune belly syndrome (4\%);

- Megacystis-microcolon-intestinal-hypoperistalsis syndrome (1\%); and

- Cloacal anomalies $(0.7 \%)^{(8)}$. Other anomalies of urethral meatus or penile foreskin such as hypospadias and phimosis due to incomplete obstruction at the distal end of urethra, rarely cause urethral dilation. Diagnosis can be made on ultrasound by careful inspection of the penis shape and urethral meatus ${ }^{(2)}$.

\section{Conclusion}

In summary, congenital megalourethra is a rare condition with poor prognosis. Isolated cases without severe associated anomalies may have better perinatal outcomes, however long-term life quality is still compromised due to severe sequelae. Along with early diagnosis, ultrasound also provides helpful data for clinicians to prognose, consult and decide the optimal treatment modality for each case.

\section{Conflict of interest}

Authors do not report any financial or personal connections with other persons or organizations, which might negatively affect the contents of this publication and/or claim authorship rights to this publication.

\section{References}

1. Moaddab A, Sananes N, Hernandez-Ruano S, Werneck Britto IS, Blumenfeld Y et al.: Prenatal Diagnosis and Perinatal Outcomes of Congenital Megalourethra: A Multicenter Cohort Study and Systematic Review of the Literature. J Ultrasound Med 2015; 34: 2057-2064.

2. Promsonthi P, Viseshsindh W: Case report and review: prenatal diagnosis of congenital megalourethra. Fetal Diagn Ther 2010; 28: 123-128.

3. Benacerraf BR, Saltzman DH, Mandell J: Sonographic diagnosis of abnormal fetal genitalia. J Ultrasound Med 1989; 8: 613-617.

4. Amsalem H, Fitzgerald B, Keating S, Ryan G, Keunen J, Pippi Salle JL et al.: Congenital megalourethra: prenatal diagnosis and postnatal/ autopsy findings in 10 cases. Ultrasound Obstet Gynecol 2011; 37: $678-683$.

5. Tonni G, Vito I, Ventura A, Grisolia G, De Felice C: Fetal lower urinary tract obstruction and its management. Arch Gynecol Obstet 2013; 287: 187-194.

6. Lissauer D, Morris RK, Kilby MD: Fetal lower urinary tract obstruction. Semin Fetal Neonatal Med 2007; 12: 464-470.

7. Sepulveda W, Elorza C, Gutierrez J, Vasquez P, Castro V: Congenital megalourethra: outcome after prenatal diagnosis in a series of 4 cases. J Ultrasound Med 2005; 24: 1303-1308.

8. Taghavi K, Sharpe C, Stringer MD: Fetal megacystis: A systematic review. J Pediatr Urol 2017; 13: 7-15. 